\title{
Berliac y el gaijin gekiga. \\ Una perspectiva mangaesca
}

\section{Berliac and gaijin gekiga. \\ A mangaesque perspective}

\section{José Andrés Santiago Iglesias}

Universidade de Vigo

José Andrés Santiago Iglesias es artista plástico-visual, profesor e investigador postdoctoral distinguido (PID) en la Facultad de Bellas Artes de Pontevedra (Universidade de Vigo). Doctor en Bellas Artes - y Premio Fin de Carrera- es, desde el año 2005, miembro del grupo de investigación dx5, especializado en gráfica contemporánea de campo expandido. Director de la línea de «Estudios Transdisciplinares sobre Cómic (ETC)» e I.P. del proyecto ministerial homónimo (ref. PID2019108898GA-I00), su investigación se centra en el análisis del manga y anime desde un enfoque estético y material. Becario de la Japan Foundation's Japanese Studies Program (2012), investigador posdoctoral invitado en la Kyoto Seika University (Japón, 20142016) y miembro de la ACDCómic desde su fundación. Es, asimismo, autor del libro Manga. Del cuadro flotante a la viñeta japonesa (Comanegra, 2010) y coordinador de la guía Japón para Otakus (Diábolo, 2018).

Fecha de recepción: 3 de octubre de 2020

Fecha de aceptación definitiva: 12 de noviembre de 2020 


\title{
Resumen
}

Este artículo se centra en las dinámicas existentes entre diferentes «sellos» de estilo manga así como al concepto de lo mangaesco, empleando como caso de estudio la novela gráfica Sadbøi (2017), del artista argentino Berliac. Se estructura en tres partes: primero, se introduce brevemente la dicotomía entre «manga como estilo» $\mathrm{y}$ «manga made in Japan»; a continuación, se exponen algunos ejemplos en los que «manga» se emplea como sello o etiqueta estilística. Por último, se analizan los aspectos mangaescos en la obra de Berliac, y la marca distintiva con la que el propio autor define su narrativa gráfica: gaijin gekiga.

Palabras clave: Berliac, gekiga, manga, mangaesco.

\begin{abstract}
This paper addresses manga from a transcultural scope - paying special attention to the interplay and dynamics of different manga labels-, discussing the role of the mangaesque through the latest graphic novel by Argentinian comic artist Berliac, Sadbøi (2017). In order to do so, this paper falls into three major sections. Firstly, I will briefly introduce the dichotomy of «manga as style» and manga as «made in Japan». Secondly, I will highlight some cases where «manga» is used as a label, especially in relation to Berliac's approach. Finally, I will address Berliac's mangaesque comics and his own signature label: gaijin gekiga.
\end{abstract}

Keywords: Berliac, gekiga, manga, mangaesque.

\section{Cita bibliográfica}

Santiago Iglesias, J. A. «Berliac y el gaijin gekiga. Una perspectiva mangaesca», en CuCo, Cuaderno de cómic n.o 15 (2020), pp. 31-51. 
Todas sus historias aluden a esa angustia urbana y postindustrial que autores como $\mathrm{Ha}$ yashi, pero también los hermanos Tsuge, Abe, Matsumoto y Tatsumi, entre otros, exploran en sus obras. Historias de un realismo profundo y dramático (acercándose al significado etimológico de gekiga), atento a las posibilidades que se escapan por los márgenes de la fortuna económica y social, y que advierten con ello de que las posibles formas de felicidad no son, en absoluto, universales. [...] Este es un caso clarísimo de absorción completa de una influencia para trabajar ese mismo territorio — ¿se podría hablar de exorcismo, a través del ejercicio de la influencia? - Es como si Berliac vistiese la piel de un autor de gekiga para comprender qué tipo de narrativas podría llevar a cabo. ${ }^{1}$

Este artículo aborda el manga desde una perspectiva transcultural, centrándose en el rol de lo mangaesco ${ }^{2}$ a través de Sadbøi, ${ }^{3}$ la novela gráfica del autor argentino Berliac. Se presta especial atención a la interacción y dinámicas existentes entre las diferentes denominaciones de manga, un análisis estético y formal de Sadbøi, así como los elementos mangaescos de esta novela gráfica. Para ello, el presente artículo se estructura en tres secciones bien diferenciadas. En primer lugar, se presentará de forma breve la dicotomía existente entre la concepción de manga como estilo y manga como made in Japan. En segundo lugar se presentarán algunos ejemplos en los que manga se emplea como denominación, especialmente a la hora de compararlos con el enfoque adoptado por Berliac. Por último, se analizarán los cómics mangaescos de Berliac y su sello característico - gaijin gekiga - con el que el autor define sus propias narrativas gráficas.

La noción de mangaesco que se emplea a lo largo del artículo es similar a la de Berndt (mangaesque). Tal y como ella lo define, mangaesco es:

Aquello que pasa como «típicamente manga» entre los consumidores habituales de este tipo de medios [...] Sin embargo, este enfoque que denomino mangaesco, entendido como «similar al manga» o «típicamente manga» — que, por supuesto, no es un término consolidado a nivel académico - permite, por un lado, llamar la atención sobre discursos populares relevantes en un contexto práctico, y, por otra parte, poner el acento sobre reflexiones teóricas, fundamentadas con criterio, sobre lo que podría o no esperarse del manga. ${ }^{4}$

Por tanto, se emplea aquí la voz mangaesco para referirse a algunos cómics -o algunas características formales de dichos cómics- porque permite ofrecer una

\footnotetext{
${ }^{1}$ Moura, P. «Desolation.Exe. Berliac (Low Spirits)», en Lerbd, 21 de junio de 2016. Disponible en: lerbd.blogspot.com/2016/06/desolationexe-berliac-low-spirits.html

2 Berndt,J. «Facing the Nuclear Issue in a 'Mangaesque'Way: The Barefoot Gen Anime», en Cinergie n. 2 (2012), pp. 148-162.

${ }^{3}$ Berliac. Sadbøi. Barcelona, Roca Editorial (Sapristi Cómics), 2017.

4 Berndt, J. Op. cit., p. 149
} 
visión muy clara de los elementos de aspecto manga presentes en ellos, enfatizando, de este modo, la autonomía del medio del manga sin depender tanto de sus raíces japonesas.

\section{Manga como estilo. Manga y sus raíces japonesas}

Cuando se intenta contextualizar y definir qué es el manga, especialmente desde una óptica internacional, se suelen presentar dos enfoques diferentes y -aparentemente - irreconciliables: el manga como un producto enraizado en Japón (producido en Japón para una audiencia japonesa y serializado en revistas periódicas); y el manga como un estilo (un tipo de arte secuencial, caracterizada por un conjunto de elementos formales altamente reconocibles y estandarizados) con complejas implicaciones dentro de un marco transcultural más amplio. Para algunos aficionados e investigadores académicos estas dos posturas parecen ser irreconciliables e incompatibles, dando como resultado un intenso debate acerca del manga y el concepto de lo mangaesco. Aquellos que apoyan la noción del manga arraigado al «made in Japan» entienden el manga como cómics japoneses - ligando inexorablemente este medio a Japón- y que cualquier narrativa gráfica producida fuera de Japón no puede, por tanto, ser considerada manga. En cambio, muchos autores de obras mangaescas que residen fuera de Japón enfatizan la dimensión formal y entienden el manga como un estilo compuesto por un conjunto de características altamente reconocibles que pueden, de facto, ser adoptadas y asimiladas como propias y ser, así, usadas para sus propias creaciones. Bajo esta definición, dichas obras serían, pues, manga, independientemente del país de creación. El primer punto de vista está conectado, en buena medida, con las tesis del origen milenario del manga, de acuerdo a las cuales dicha genesis se remontaría al siglo xiII y a los Chojjügiga los rollos ilustrados de las aves y los animales - y otros emakimono, las xilografías Ukiyo-e del período Edo y el Hokusai Manga (1814-1878), trazando así una cierta continuidad con el manga moderno. No obstante, este enfoque ha sido ampliamente cuestionado, ${ }^{5}$ en especial por la ausencia de una continuidad real más allá de las simple diacronía, ya que el origen del manga no se puede entender ${ }^{6}$ sin las tiras cómicas y semanarios satíricos europeos y estadounidenses de finales del siglo XIX y principios del xx. ${ }^{7}$

\footnotetext{
5 Berndt, J. «Traditions of Contemporary Manga (1): Relating Cómics to Premodern Art», en Signs. Studies of Graphic Narratives, n. ${ }^{\circ} 1$ (2007), pp. 33-47.

${ }^{6}$ Berndt, J. «Manga and 'Manga': Contemporary Japanese Comics and their Dis/similarities with Hokusai Manga», en Jablons KI, A. et al. (eds.) Civilization of Evolution, Civilization of Revolution, metamorphoses in Japan 1900-2000. Cracovia, Museum of Japanese Art and Technology, 2007, pp. 210-222.

7 Stewart, R. «Manga as schism: Kitazawa Rakuten's resistance to 'Old-Fashioned'Japan», en Berndt, J. y Kümmerling-Meibauer, B. (eds.) Manga's Cultural Crossroads. Londres, Routledge, 2013, pp. $27-49$.
} 
Un ejemplo claro de esta clasificación dicotómica puede verse en la definición de la palabra manga, recogida en el diccionario de la lengua española desde el año 2012. En esa fecha, la Real Academia Española decidió incluir esta voz japonesa como parte del léxico español. Sin embargo, la satisfacción inicial tanto de los especialistas como de los aficionados a este medio se convirtió en un clamor de desaprobación cuando se conoció la definición recogida por dicho organismo: «género de cómic de origen japonés, de dibujos sencillos, en el que predominan los argumentos eróticos, violentos y fantásticos». ${ }^{8}$ Esta descripción calcaba, en buena medida, otras fórmulas similares en diccionarios de la lengua inglesa, como el Oxford Dictionary en la época. Las protestas de fans, editores y autores (incluso desde la embajada japonesa en España) motivaron un cambio en la definición por parte de la RAE, optando por una fórmula mucho más neutra, aséptica y carente de matices: "Cómic de origen japonés" ("Manga")». Este incidente resulta revelador por cuanto pone de manifiesto el debate acerca de lo mangaesco que todavía está presente en este medio. Asimismo, y a pesar de que la primera definición es claramente peyorativa, ambas fórmulas proporcionan una visión bastante amplia e inclusiva de qué se entiende por manga para una mayoría de lectores en todo el mundo. La primera definición pone el énfasis en aspectos formales y temáticos, mientras que la segunda - e imperante en la actualidad - acentúa el carácter japonés de este medio gráfico. Se podría decir, incluso, que esta dicotomía se puede entender dentro de la reciente campaña de promoción cultural Cool Japan, al igual que sucede con el anime y otros medios, evidenciando la dificultad de reconciliar la idea de manga como producto genuinamente japonés con la clara dimensión transnacional de lo mangaesco, que en las últimas dos décadas no ha dejado de crecer y transformarse.

El Dr. Zoltan Kacsuk abordó esta dicotomía entre estilo y origen en una ponencia dentro del la 15. a Conferencia Anual Internacional de Estudios Japoneses celebrada en Manila en el año 2015, y que desarrolló con mayor profundidad en un artículo de obligada lectura publicado en la revista Arts. ${ }^{9}$ La tendencia que propugna la comprensión del manga como un estilo gráfico se centra en aspectos visuales y de contenido, poniendo el foco en un conjunto de elementos muy reconocibles, como los diseños altamente convencionalizados de personajes y fondos, los códigos visuales, el diseño de páginas, los géneros demográficos, los ejes temáticos, los tropos visuales, el predominio de un punto de vista subjetivo o las estructuras narrativas, entre otros muchos. En cambio, aquellos que defienden el manga como un producto atado irremediablemente a su origen japonés suelen resaltar aspectos productivos o de la industria editorial japonesa, como el modelo de serialización en revistas periódicas

8 Abella, A. «La definición de la RAE para 'manga' indigna al mundo del cómic», en El Periódico, 22 de junio de 2012. Disponible en: www.elperiodico.com/es/ocio-y-cultura/20120622/la-definicionde-la-rae-para-manga-indigna-al-mundo-del-cómic-1961941

9 Kacsur, Z. «Re-Examining the 'What is Manga' Problematic: The Tension and Interrelationship between the Style Versus Made in Japan Positions», en Arts, Volume 7, Issue 3 (2018). doi: 10.3390/ arts7030026. 
mangashi, los mecanismos de circulación y consumo, el papel indispensable de los editores japoneses en el proceso creativo, el entorno visual y cultural japonés indudablemente presente en muchas de las obras, o el potencial media-mix del manga como medio. Para muchos lectores, el manga no es tan solo un género de arte secuencial, sino una conexión directa con Japón y, por ello, Japón persiste como garante y fuente que legitima la singularidad del manga. En realidad, el hecho de que a menudo se plantee este debate dicotómico entre manga como un estilo y manga como producto japonés no significa, en modo alguno, que ambas nociones sean irreconciliables, dado que un enfoque híbrido es perfectamente posible. Kacsuk explica cómo ambas facetas están, en realidad, profundamente interrelacionadas. El manga no puede ser entendido únicamente como medio visual, sino también como industria - con sus mecanismos únicos de producción y consumo- y como generador de dinámicas interpersonales entre lectores y aficionados. Históricamente, los aspectos productivos han conllevado profundas transformaciones estéticas, al tiempo que la forma y materialidad del manga han condicionado, de igual modo, el actual modelo productivo.

\section{E1 manga como movimiento}

La diferenciación dicotómica discutida en párrafos anteriores ha centrado - $\mathrm{y}$ sigue centrando - una parte importante del debate sobre el manga, tanto a nivel académico como entre aficionados a este medio. Sin embargo, hay muchas maneras de entender el manga, más allá de esta sencilla polarización. Casey Brienza ${ }^{10}$ planteó una cuantas posibilidades a la hora de definir la etiqueta manga: manga como estrategia de márquetin, manga como estilo, manga como producto japonés, manga como una cualidad, y lo que ella denominó «¿de verdad te importa tanto?» («Why do you care so much», pero que bien se podría traducir como «y qué más da»). Pero también se podrían incluir como concepciones igualmente válidas la del manga como reivindicación, el manga como identidad, o incluso el manga como una mezcla de todas estas tipologías. En cierto modo, lo que el debate dicotómico estilo vs. Japón no ha sido capaz de entender es que el manga también se emplea tanto por autores como por comunidades de aficionados en todo el mundo, no cómo estilo ni como algo anclado en Japón, sino como un movimiento con una enorme base de seguidores, con un profundo sentido de pertenencia y un conjunto de valores simbólicos e intangibles más profundos. Más allá de las connotaciones formales y estilísticas, el manga como movimiento se construye en torno a la idea de comunidad, ya que la comunidad es lo que en última instancia define la dimensión y el alcance de esta noción de manga. No en vano, los autores mangaescos se apoyan en otros creadores afines, así como en la comunidad de aficionados, cuando formulan sus reivindicaciones artísticas.

10 Brienza, C. «Manga is not Pizza: The performance of ethno-racial authenticity and the politics of American anime and manga fandom in Svetlana Chmakova's Dramacon», en Brienza, C. (ed.) Global Manga: "Japanese" Cómics without Japan? Londres, Ashgate, 2015, pp. 95-113. 
En las últimas décadas, la noción de manga entendido como estilo y como un movimiento se ha multiplicado en todo el mundo, suscitando nuevos interrogantes acerca de la materialidad del medio, sus profundas conexiones con los aspectos editoriales y productivos, o el rol de lo mangaesco. En algunos casos, ha habido propuestas para unificar algunas de estas corrientes bajo diferentes sellos y movimientos estilísticos (nacionales, culturales, generacionales, etc.) —como la Nouvelle Manga, el manfra, el iberomanga, el OEL manga, o el gaijin gekiga de Berliac, entre otros muchos- por motivos de lo más dispares: como una estrategia de comercialización, para la promoción de la propia obra, o incluso arraigado a cuestiones de identidad y autoría. Es por ello que muchas obras etiquetadas como OEL manga, Manfra o Iberomanga - pero también cómics con origen en Filipinas, Indonesia, Vietnam o Brasil- comparten rasgos vinculados a la noción de manga como estilo y como movimiento.

Sin embargo, a veces los límites de lo mangaesco resultan más difusos, más complejos y difíciles de definir, en especial cuando se trata de la obra de artistas con un importante componente transnacional como el hispano-japonés Ken Niimura. Niimura es conocido por su dibujo en la novela gráfica I Kill Giants, ${ }^{11}$ ganadora de numerosos premios internacionales, o la serie de manga Henshin (2013), publicada de manera periódica en la revista Ikki y recopilada posteriormente en un volumen tankōbon. El dibujo de Niimura se adapta para ajustarse a los requisitos específicos de cada mercado (formato, tamaño, velocidad y dirección de lectura, etc.) pero manteniendo muchos rasgos comunes, y un estilo visual y narrativo altamente reconocible, con independencia de si su trabajo se publica en Estados Unidos (como cómics), en España (como novela gráfica) o en Japón (como manga). Tanto en I Kill Giants como en Henshin se da un tratamiento similar en la disposición de las viñetas, los diseños de personajes o la modulación de la línea —ágil y sin refinar- sin que se evidencie (más allá del lógico sentido de lectura) si se trata de un cómic estadounidense o un manga japonés.

Dentro del potente mercado francés, hay dos casos que adoptan dos enfoques radicalmente opuestos frente al fenómeno mangaesco: El grupo de artistas de la Nouvelle Manga - liderados por Frédéric Boilet- y la serie Lastman12 creada por Bastien Vivès, Balak y Michaël Sanlaville. Francia es un país donde el concepto de autor y autoría está profundamente arraigado. En lo que al manga se refiere, el caso francés resulta muy interesante, por sus enormes contradicciones. No deja de ser significativo que, siendo Francia el segundo mercado del manga más potente en todo el mundo, solo por detrás de Japón, también haya sido uno de los países más proteccionistas y con una industria nacional más beligerante para con el cómic japonés.

${ }^{11}$ I Kill Giants (en su edición en España, Soy una matagigantes) es obra del guionista Joe Kelly y el dibujante J. M. Ken Niimura, publicada originalmente por Image Cómics en 2008. I Kill Giants ganó el Gold Award en el 5th International Manga Award de 2012, y fue nominada a un premio Eisner en la categoría de Mejor Obra para Adolescentes. En el año 2017 fue adaptada a un largometraje por Anders Walter.

12 Publicada en Francia, por la editorial Casterman, entre los años 2013 y 2019. 
La Nouvelle Vague Manga es una denominación acuñada por Kiyoshi Ksumi, editor de la revista Cómickers, para referirse a los cómics de Frédéric Boilet, que este tomó para sí. «Boilet consideró que el nombre englobaba a la perfección las pretensiones para con la vida cotidiana que intentaba plasmar en sus historias, por lo que decidió hacerla suya reduciéndola a Nouvelle Manga». ${ }^{13}$ En el año 2001, el propio Boilet publicó su manifiesto de La Nouvelle Manga, reivindicando un nuevo espacio a medio camino entre la bande dessinée francesa y el manga japonés, uniendo — en opinión del autor- lo mejor de ambos mundos. No obstante, el manifiesto de La Nourvelle Manga de Frédéric Boilet no está exento de polémica. En opinión de este autor, las historias habituales en el cómic europeo son infantiles y donde abundan los guiones simplones y argumentalmente poco elaborados. En cambio, destacan por su dibujo de calidad y trazo sofisticado, deudor de la tradición artística del continente. Al mismo tiempo, Boilet contrapone estas afirmaciones con lo que, a su parecen, domina el mercado del manga: historias cotidianas y de gran desarrollo argumental y emocional, pero caracterizadas por un dibujo de baja calidad. La opinión de Boilet resulta, tanto en lo referente a la la bande dessinée como al manga, excesivamente superficial, ya que fuerza una lectura que no responde a la realidad para poder construir su relato.

Los artistas englobados bajo el sello de la Nouvelle Manga ahondan en la temática costumbrista y el retrato de lo cotidiano, que el autor francés ensalzó en su manifiesto teórico. En él, Boilet pone el acento sobre las conexiones de su manga con la estética de la nouvelle vague, así como con el manga d'auteur, del que Jiro Taniguchi —un autor que históricamente se ha movido al margen del modelo industrial del manga y sus mecanismos de serialización- terminó por convertirse en referente.

\footnotetext{
Además de «el» manga, esencialmente, cómics japoneses para un público compuesto en su mayoría por adolescentes, está «la» manga, haciendo referencia a los cómics japoneses de autor, adultos y universales, que hablan de hombres y mujeres y su vida cotidiana: una manga más cercana a, por ejemplo, las películas de Yasujirô Ozu y Jacques Doillon o a las novelas de Yasushi Inoue que a Sailor Moon o Luc Besson. ${ }^{14}$
}

Sin embargo, para muchos lectores las obras de la Nouvelle Manga distan mucho de los que un manga a la europea debería ser. En cierto sentido, la Nouvelle Manga también ocultaba una intención claramente comercial; una marca que aunase bajo un mismo sello un conjunto de trabajos de autores independientes. A fin de cuentas, la perspectiva autoral se ha empleado a menudo con una doble función; como reivindicación artística, pero también como estrategia puramente comercial (similar, en este

13 Romero Leo, J. «La estética de la Nouvelle Manga. La reivindicación de lo cotidiano en la nueva novela gráfica franco-japonesa», en CuCo, Cuadernos de cómic n. ${ }^{o} 8$ (2017), pp. 88. Disponible en: http://cuadernosdecomic.com/docs/revista8/La estetica de la Nouvelle Manga.pdf

${ }^{14}$ Boilet, F. «Manifiesto de la Nouvelle Manga», en Boilet.net, 2001. Disponible en: http://www. boilet.net/es/nouvellemanga manifeste $2 . h \mathrm{tml}$ 
caso, a la propia nouvelle vague). El movimiento impulsado por Boilet permitió un mayor diálogo entre artistas franceses y japoneses, pero la verdad es que muchas de las obras amparadas bajo esa marca apenas se asemejan a nada remotamente mangaesco, ni desde un punto de vista del estilo, ni en lo tocante a sus aspectos productivos. De un modo similar a lo que sucedió con el japonismo y los impresionistas, la denominación manga juega un papel muy importante para la Nourvelle Manga en tanto que herramienta de márquetin, puesto que este movimiento artístico también tiende hacia una estetización del manga sin ahondar en un conocimiento más profundo del medio. Cabe preguntarse, pues, si la Nouvelle Manga no es tanto una revolución, como un inteligente estrategia de promoción para subirse al fenómeno imparable de la novela gráfica en Europa.

\section{Manga à la mode}

El caso de Lastman es peculiar, por cuanto no imita los principales rasgos estéticos y características formales del manga, sino que absorbe y reinterpreta aspectos productivos y de promoción típicos de este medio. En varias ocasiones, sus autores han definido Lastman como un «manga de estilo francés», ${ }^{15}$ con una gran influencia de grandes títulos como Dragon Ball (1984) de Akira Toriyama, Berserk (1989) de Kentaro Miura, o El Puño de la Estrella del Norte (1983) de Buroson y Tetsuo Hara, pero también de otras referencias de la cultura popular como las películas de Disney, algunas series televisivas de los últimos años, las franquicias de películas de superhéroes, los filmes de acción de Sylvester Stallone, o los blockbusters de la década de los ochenta y noventa del conocido director Steven Spielberg. Tal y como explica Vivès, «usamos el formato y algunos códigos del manga pero no estamos jugando a imitar la cultura japonesa», ${ }^{16}$ al tiempo que elogia la eficiencia del sistema editorial japonés en el exigente desarrollo de una serie extensa como es el caso de Lastman. En realidad, Lastman se inspira en el manga como producto, en aspectos sutiles - pero de vital importancia en su percepción por parte de los fans- como sus volúmenes de tamaño kanzenban y sobrecubierta, o el empleo del color en las primeras páginas de cada volumen, monocromático en el resto. Al igual que el manga, esta serie explora su potencial media-mix con productos como la serie animada a modo de precuela, los video-juegos, o los productos de merchandising. A priori, una obra como Lastman no debería encajar dentro de lo mangaesco (máxime si se compara con obras catalogadas bajo la denominación manfra), pero el volumen, en tanto que objeto, sí presenta claras reminiscencias a un tomo comercial japonés. El precio en Francia y España, similar al de obras japonesas de similar tamaño y acabado, contribuye a mantener la ilusión.

\footnotetext{
15 Brunner, V. «Lastman, le manga remixé french touch» en Les Inrockuptibles, 1 de mayo de 2014. Disponible en: www.lesinrocks.com/2014/05/01/livres/lastman-manga-remixe-frenchtouch-11501782/

16 Idem.
} 
Lastman es una obra colectiva, entendida esta no en el sentido de las producciones mainstream estadounidenses (con guionista, dibujante, entintador o colorista), sino donde los tres autores están involucrados en todas las fases del proceso creativo. Los propios artistas han explicado en varias ocasiones los detalles de su trabajo en equipo y cómo cada aspecto del mismo es compartido con precisión. Vivès define las líneas maestras de la historia, la creación de los personajes y el desarrollo general de la acción, que discute con sus dos colegas. Balak se encarga de estructurar la narración en arcos, volúmenes y capítulos, así como de planificar todo el storyboard y la división en viñetas en cada página. Finalmente, Vivès y Sanlaville se encargan del dibujo, a razón de unas veinte páginas por semana. ${ }^{17}$ Aun con notables diferencias —en especial a la hora de conceder la autoría - el proceso de trabajo descrito por los tres artistas no difiere tanto del que el mangaka, su editor y los ayudantes emplean en el desarrollo de un manga comercial; incluso el ritmo de trabajo es, en muchos aspectos, similar al de una obra seriada en una revista mangashi con periodicidad semanal.

Sin embargo, aunque Vivès, Balak y Sanlaville afirman, a menudo, estar dibujando un manga francés, ${ }^{18}$ parece bastante evidente que en la obra predomina el enfoque autoral; a excepción de algunas transiciones entre viñetas que podrían recordar a una composición más mangaesca, lo cierto es que Lastman no emplea otros elementos distintivos que se asocian habitualmente con el «estilo manga». Al mismo tiempo, el uso de una actriz porno japonesa Hitomi Tanaka como parte de la campaña promocional de la serie - con la problemática que ello conlleva, desde el uso de tópicos ofensivos hasta la hipersexualización de la mujer japonesa-, invita a reflexionar sobre cuantas de esas referencias procedentes del manga, a menudo esgrimidas por los autores, son reales; esto es, si corresponden a una calculada estrategia de márquetin o, por el contrario, son solo fruto de un oscuro y sarcástico sentido del humor.

\section{Sadbøi}

En el año 2016, el artista argentino Berliac presentó la novela gráfica Sadbøi, que narra la historia de un refugiado (Sadbøi) que llega a un país escandinavo, ${ }^{19}$ donde debe lidiar con diferentes situaciones - fruto de su estatus irregular así como de las expectativas sociales que se generan a su alrededor - y que finalmente crece hasta convertirse en un delincuente. En esta época de la post-verdad, Sadbøi se erige como

${ }^{17}$ LE SAux, L. «BD : Lastman, un manga à la française de Vivès, Balak et Sanlaville», en Télérama, 29 de mayo de 2013. Disponible en: www.telerama.fr/livre/bd-lastman-un-manga-a-la-francaise-devives-balak-et-sanlaville,96613.php

18 Brunner, V. Op. cit.

19 Aunque el país de destino no se especifica en la obra, en diferentes entrevistas Berliac aclaró que la historia podría tener lugar en Suecia. El título — Sadbøi - alude a Sad Boys, el grupo de música sueco liderado por el actor y rapero Young Lean. 
una crítica sólida e inteligente frente a los discursos anti-inmigrantes, al tiempo que proporciona un análisis perspicaz del dilema de la identidad personal y cuestiona muchos de los fundamentos del arte contemporáneo y la performance. Sadbøi fue definida como «una declaración sobre el trato a los inmigrantes - el desafío que supone que se espere de ellos el ser capaces de ajustarse a los ideales de una determinada sociedad en un mundo que condena prematuramente a los extranjeros». ${ }^{20} \mathrm{El}$ posicionamiento de Berliac frente al debate en torno a la inmigración es rico y complejo, sin caer en respuestas fáciles. Berliac señala los verdaderos desafíos a los que se enfrenta un niño inmigrante cuando llega a un país extranjero, que no se reducen al simple hecho de entrar en el país, sino a la soledad que le sigue, a su inserción en un entorno potencialmente hostil, a los problemas de adaptación y la falta de apoyo y seguimiento en su desarrollo personal o a las expectativas de aquellos que le rodean y que a menudo eligen ignorar el sórdido pasado personal que le empujó a huir de su hogar en un primer lugar. El propio Berliac afirma: «Me doy cuenta de que su condición de refugiado lleva implícita una identidad impuesta por terceros». ${ }^{21}$ En cierta medida, Berliac muestra que la cuestión de los refugiados no se resuelve con un brote puntual de solidaridad, sino que requiere — al menos inicialmente - comprensión y un plan a largo plazo. Además, su acercamiento paródico a la industria del arte contemporáneo - a menudo representada como llena de esnobismo y postureo- oculta una consideración más profunda de la noción de apropiación en el arte contemporáneo, el uso de la performance como práctica artística (incluyendo la brillante despedida de Sadbøi a modo de atraco-performance) o la hipocresía del mercado del arte. Así pues, Sadbøi «examina el papel del arte en el desarrollo de la propia identidad, al tiempo que desafía al lector con preguntas directas y francas». ${ }^{22}$ Mostrando violencia, drama y un oscuro sentido del humor - bien equilibrado- Sadbøi es la novela gráfica más destacada de Berliac hasta la fecha, y el mejor ejemplo de una narración y un estilo visual únicos, etiquetados por el propio Berliac como gaijin gekiga.

Sin embargo, mientras que la publicación de Sadbøi vino acompañada de las buenas reseñas de la crítica, esta no estuvo exenta de polémica, con feroces críticas por parte de algunos lectores y prensa digital, relacionados tanto con algunas de sus declaraciones como con el enfoque mangaesco de su obra. El hecho de presentar su trabajo bajo la etiqueta de gaijin gekiga ha despertado la ira de aquellos lectores que - tal y como se explicó en los párrafos anteriores- niegan la posibilidad de que se haga manga fuera de Japón. Más aún, y tal y como el propio Berliac explicó en el curso de una conversación

${ }^{20}$ Burns, P. «An Apology», en Drawn E Quarterly, 2 de junio de 2017. Disponible en: http://www. drawnandquarterly.com/blog/2017/06/apology

${ }^{21}$ Parkas, V. «Berliac, el dibujante de cómic que reivindica el robo cultural como esencia creativa», en PlayGround, 14 de noviembre de 2017. Disponible en: http://www.playgroundmag.net/ $\underline{\text { lit/Berliac-el-chico-triste-que-hizo-de-la-apropiacion-cultural-una-forma-de-empoderamien- }}$ to $23343930 . \mathrm{html}$

22 Idem. 
personal en el Festival de Cómic de Estocolmo en 2018, a medida que su trabajo ha ido evolucionando hacia un estilo cada vez más mangaesco, también ha empezado a recibir reproches por parte de autores y críticos de su país natal, por desviarse de los que ellos entienden como estética underground (tal y como explicaba Berliac, durante su infancia el manga se asociaba con lo femenino y, por tanto, el estilo mangaesco es visto como poco masculino e impropio de autores varones). ${ }^{23}$ La mayor controversia surgió a raíz de la cancelación de la publicación de Sadbøi en Estados Unidos, por parte de Drawn $\&$ Quarterly, después de que numerosos lectores y la escritora trans Sarah Horrocks acusasen a Berliac de transfobia. ${ }^{24} \mathrm{El}$ incidente se produjo tras una discusión en redes sociales con la propia Horrocks acerca de un artículo titulado «Gay-jin», ${ }^{25}$ publicado por Berliac en Internet en el año 2015, y en el que comparaba el proceso de conversión de un dibujante no japonés en mangaka con la transición de género. Curiosamente, y lejos del debate original, esta discusión derivó en acusaciones de apropiación cultural, que Berliac abordó con grandes dosis de sarcasmo en «Manga is masturbation»; ${ }^{26}$ el último capítulo de la serie de Asian Store Junkies (2017), una colección de historias cortas sobre dos amigos y compañeros de piso fascinados con el umami (una de las categorías de sabor —además del dulce, salado, ácido y amargo - asociado habitualmente a los glutamatos, en especial el glutamato monosódico).

\section{Berliac y el gaijin gekiga}

Gekiga es una voz japonesa que significa, literalmente, «dibujos dramáticos». Normalmente se emplea para definir un estilo de manga dirigido al público adulto, caracterizado por el amplio uso de la perspectiva y encuadres cinematográficos ${ }^{27}$ y temas maduros, y nacido como respuesta a las narrativas gráficas de corte más infantil, como las obras tempranas de Osamu Tezuka u otros autores que dominaron la industria del manga a finales de los años cuarenta y principios de los cincuenta. El nombre gekiga fue acuñado en 1957 por el ahora célebre mangaka Yoshihiro Tatsumi y adoptado por otros dibujantes japoneses con ideas afines. ${ }^{28}$ Incluso hoy en día, aunque la denomi-

${ }^{23}$ Idem.

${ }^{24}$ Parkas, V. «Unas declaraciones transfóbicas del dibujante Berliac le han valido el odio de medio internet», en PlayGround, 8 de junio de 2017. Disponible en: www.playgroundmag.net/lit/Holocausto-Berliac-transfobia-cancelaciones-editoriales 22594415.html

25 Horrocks, S. Mercurial Blonde, 21 de abril de 2015. Disponible en: mercurialblonde.tumblr.com/ post/117018318913

${ }^{26}$ Berliac. «Manga is Masturbation», en Asian Store Junkies. Vice, 2017. Disponible en: www.vice. com/en us/article/7xxdy4/manga-is-masturbation-todays-cómic-by-berliac-cómic

27 Holmberg, R. «An Introduction to Gekiga, 6970 A.D.». The Comics Journal, 24 de abril de 2011. Disponible en: www.tcj.com/an-introduction-to-gekiga-6970-a-d/3/

28 Tatsumi se refirió a Taxi Fantasma (Yürei takushii, 1957) —una de sus primeras obras- como gekiga. Desde entonces, esta voz se ha empleado para definir no solo la obra de Tatsumi, sino la de otros muchos autores con planteamientos formales y argumentales similares. Sanpei Shirato, Yoshiharu 
nación gekiga ha perdido gran parte de su significado original dentro de la industria del manga (dado que muchas de sus innovaciones formales y temáticas han sido ampliamente adoptadas) algunos autores siguen utilizándola con un claro objetivo estético o —una vez más- para diferenciarse del estilo del manga mainstream que domina la industria editorial japonesa. ${ }^{29}$

Más allá de su fascinante y sólido argumento, Sadbøi resulta interesante para este estudio porque suscita preguntas innovadoras acerca del gekiga $-\mathrm{y}$ su vigencia en el contexto actual - al tiempo que ejemplifica algunas de las contradicciones inherentes al debate manga como estilo y manga como producto japonés. Históricamente, el gekiga se caracteriza por sus diseños de personajes, alejados de los estándares que dominan el mercado del manga contemporáneo, así como un diseño de página y una disposición de las viñetas más rígida. No obstante, desde un punto de vista puramente conceptual, el seinen manga de la actualidad puede entenderse como un heredero del gekiga, dado que muchos de los rasgos definitorios que se utilizaban comúnmente para diferenciar el gekiga de obras anteriores - lectores maduros, argumento dramático y abundancia de enfoques cinematográficos- están presentes en numerosos títulos de seinen manga en la actualidad. La (voluntaria) identificación de Berliac con un género de manga arcaico — dado que los temas e innovaciones formales del gekiga han sido asimilados hace mucho tiempo por la industria editorial y están presentes, hoy en día, en el manga mainstream - es muy interesante, por cuanto proporciona una perspectiva diferente del concepto de mangaesco cuando se compara con otras marcas mangaescas. De algún modo, Berliac elige referirse a sí mismo como mangaka y a su obra como gaijin gekiga por cuestiones de identidad - para etiquetarse a sí mismo de forma diferente a otros artistas y, con ello, lograr posicionarse dentro de la gran industria del cómic, pero también para reivindicar sus cimientos mangaescos y su conexión más profunda con el medio original japonés y para definir un movimiento artístico influenciado por el manga — que gaijin gekiga no se quede únicamente en una simple etiqueta con fines estilísticos, sino que se consolide en un movimiento con su propia estrategia, definido por un ideario social y artístico - situándose, por lo tanto, en una tercera categoría que no estaría totalmente recogida en el análisis de Kacsuk.

Tsuge y muchos otros artistas, incluyendo varios de los que publicaron en la influyente revista Garo (1964) del editor Katsuichi Nagai, son considerados claros representantes del gekiga.

29 Es el caso de Yūichi Yokoyama (1967), uno de los pocos autores japoneses que, sin haber pertenecido a la generación original de autores veteranos del gekiga, emplea la voz gekiga para referirse a su propia obra. En el caso de Yokoyama — que define su trabajo como neo-gekiga - este término connota un claro distanciamiento respecto al manga mainstream. En su obra World Map Room (2013), Yokoyama escribe: «World Map Room es una obra de gekiga. Ninguno de los hombres sonríe o se ríe. Siempre son cautelosos, sospechan constantemente de las cosas a su alrededor, y hablan en un tono poco amigable. No parece que hayan venido a esta megalópolis por ocio o entretenimiento, sino por algún propósito lleno de tensión. Los hombres con los que se encuentran también sospechan de los extraños y no parecen dispuestos a tratarlos con amabilidad». Aunque no exenta de teatralidad, la descripción de Yokoyama conecta con muchos de los supuestos originales de Tatsumi. 
La marca gaijin gekiga esconde un gran potencial, aunque inicialmente presente las mismas contradicciones comunes a otros artistas internacionales de influencia mangaesca. A menudo los autores de OELmanga, manfra, amerimanga, iberomanga y muchos otros movimientos similares enfatizan la importancia de lo japonés en sus obras, pero no las perciben como tenedoras de un estilo diferente (inicialmente derivativo). Paradójicamente, al subrayar el vínculo ideológico de sus narraciones gráficas con el origen japonés en el plano discursivo, estos artistas se alejan del carácter transnacional que, en última instancia, habría resuelto la problemática de la «autenticidad». ${ }^{30} \mathrm{Al}$ definirse a sí mismo como mangaka y calificar su obra como gaijin gekiga, Berliac parece socavar los mismos principios que persiguen sus cómics, esto es: enfatizar la dimensión transnacional del manga frente a un discurso japonocéntrico. En apariencia, la fórmula gaijin gekiga acarrea una difícil contradicción: gaijin (lit. extranjero, forastero) se distancia inmediatamente de la raíz japonesa original del manga, mientras que gekiga — debido a lo obsoleto del término- es sustancialmente distinto de cualquier movimiento contemporáneo de manga. Berliac ha abordado repetidamente esta contradicción en sus entrevistas:

\begin{abstract}
Yo no estoy influenciado por el manga: yo hago manga [...] Si de alguien va a un dojo de kárate, con uniforme de kárate, y utilizando terminología de kárate, no decimos que está influenciado por el kárate, sino que hace kárate, no entiendo por qué alguna gente sigue negándose a admitir que yo sea un mangaka. ${ }^{31}$
\end{abstract}

Con esa afirmación, Berliac parece confirmar su apuesta por el enfoque «manga como estilo». El uso de diseños de personajes y distribución de viñetas similares a los utilizados por los primeros autores de gekiga, así como algunos códigos visuales y expresiones faciales utilizados en el manga contemporáneo serían suficientes, a ojos del artista argentino, para posicionar su obra como un manga y, por lo tanto, definirse como mangaka. Si este fuera el caso, bastaría por lo tanto con crear obras con una estética mangaesca para presentarse como un mangaka. Sin embargo, tal y como sucede con ese tipo de obras mangaescas, Berliac ignora deliberadamente los aspectos productivos de la industria del manga en el Japón —las publicaciones periódicas en revistas seriadas o el papel del editor en el proceso creativo- como rasgos determinantes para convertirse en un mangaka. No obstante, al explicar con mayor detalle la idea que subyace bajo el sello gaijin gekiga, Berliac desvela un enfoque bastante más interesante y diferenciador. Al empaparse de un movimiento de manga arcaico - $\mathrm{cu}^{-}$ yas innovaciones, tal y como se ha repetido, ya han sido asimiladas por la industria mainstream - Berliac no se arriesga a convertirse en una copia, ya que el gekiga está considerado como un estilo manga pasado de moda, y por lo tanto la obra de Berliac no se convierte en un derivado ni en una imitación, ya que no se desvía de ningún estilo actual. Así, mientras que algunos académicos y críticos pueden identificar las conexiones, por lo demás obvias, con la estética del gekiga, muchos lectores y aficio-

\footnotetext{
30 Kacsuk, Z. Op. cit.

${ }^{31}$ Parkas, V. Op. cit.
} 
nados al manga encontrarán el gaijin gekiga de Berliac sustancialmente diferente del manga actual, independientemente de si estos lectores están o no familiarizados con las obras de gekiga (que, a excepción de los títulos más conocidos, han sido poco traducidas en Europa y Estados Unidos) o porque perciben que la influencia del estilo visual del gekiga en las novelas gráficas de Berliac - ya que el gekiga es un género que se remonta varias décadas en el pasado- responde más a un sentido de referente y homenaje que al de la copia. Berliac explica su elección del gekiga:

Aquí subyace otra razón por la que me gusta llamar a mi propia obra Gaijin-Gekiga: si gekiga era la expresión japonesa, en forma de cómic dramático, de sus propias ansiedades colectivas en tiempos de hipercapitalismo, entonces puedo decir sin duda que el enfoque principal de mi trabajo es el mismo, solo que sobre mi propio lugar y tiempo, por supuesto. [...] El gekiga fue una forma preciosa y digna de aceptar la aplastante realidad de la derrota de la posguerra. Las narrativas occidentales de hoy en día, por el contrario, insisten en que estamos en el lado ganador, incluso cuando nuestra realidad apunta claramente a lo contrario [...]. No digo que haya dignidad en ser un perdedor, sino en ver la realidad como lo que es. Drama sin exagerar. Esa es la esencia de gekiga. ${ }^{32}$

Cuando se le pregunta acerca del gaijin gekiga, casi se espera que Berliac se centre en el discurso sobre el origen del manga, en vez de los aspectos formales, pero no es el caso. Llamándose a sí mismo mangaka y etiquetando su trabajo como gaijin gekiga, este joven autor está formulando una reivindicación al tiempo que desafía muchas de las convenciones habituales. Gaijin gekiga es, por supuesto, parte de una estrategia de márquetin, pero en absoluto es el objetivo principal que persigue el autor. El gaijin gekiga de Berliac es una marca creada para disolver la categorización hermética en los cómics, hasta que no haya más etiquetas. Berliac sostiene que el uso de ciertas referencias culturales y/o estéticas en entornos distintos de aquellos para los que fueron concebidas originalmente enriquece el discurso y derriba fronteras. ${ }^{33}$ Decía Berliac en el curso de una entrevista: « $i N o$ es esto un poco como inmigrar? Aprendo tu gramática visual con la mayor fluidez posible, para hablarte de mí, para conectarme, y en el proceso también aprendo sobre tu propia cultura». ${ }^{34}$

Berliac piensa en el cómic como un todo sin etiquetas entre los cómics de EE. UU., la $B D$ europea, la novela gráfica o el manga. Por ello, afirma que los aspectos formales habitualmente vinculados a estas etiquetas deben utilizarse independientemente del lugar de su creación, privándolos del bagaje cultural que a veces conllevan, tomando prestados únicamente los rasgos formales con una intención estética y narrativa, y do-

32 Stıvè, V. «Berliac e la scena Gaijiin Manga dell'antologia Kuš! \#25», en Fumetto Logica, 28 de febrero de 2017. Disponible en: http://www.fumettologica.it/2017/02/berliac-intervista-kus-gaijin-manga/2/

33 Parkas, V. Op. cit.

${ }^{34}$ Moneyron, J. «kuš! Aesthetics», en The Comics Journal, 27 de octubre de 2016. Disponible en: http://www.tcj.com/kus-aesthetics/ 
tándolos, en última instancia, de un nuevo significado. Además, el gaijin gekiga es un intento audaz de abordar la cuestión de la fluidez en el cómic, que a su vez facilita la idea de una identidad fluida (fácilmente permutable, de formas polifacéticas, y adoptando una perspectiva transcultural). Solo cuando se logre este objetivo de no tener etiquetas, podría Berliac renunciar a la marca gaijin gekiga, dado que, a su entender, ya no sería necesaria. Así pues, la postura de Berliac aboga, en última instancia, por un enfoque transnacional y transcultural en el cómic (independientemente de su país de origen). Afirma Berliac:

En último extremo, gaijin manga apunta menos a la nacionalidad del artista y más hacia un momento histórico en el que los límites de género y cultura, en general, están siendo erosionados a una velocidad endiablada [...]. Gaijin sirve como intermediario amistoso entre un pasado fracturado y un futuro integrador. ${ }^{35}$

Los aspectos formales en la obra de Berliac también responden a una ingeniosa estrategia visual. Por lo general, el OELmanga recurre con profusión a elementos altamente convencionalizados, como los diseños chibi; el uso de bocadillos verticales; la disposición multicapa de las viñetas, de acuerdo a una estética shöjo-mangaesca; el grafismo de las onomatopeyas e incluso el uso del lenguaje (con abundancia de expresiones japonesas cotidianas y sufijos honoríficos). Sin embargo, a la hora de definir su estilo, Berliac se fija en los primeros autores del gekiga, muy diferentes del manga mainstream contemporáneo. Los movimientos corporales y el diseño general de los rostros en los personajes de Berliac remiten inmediatamente a los de las primeras obras de Tatsumi, a los rasgos muy expresivos de los héroes de Shōtarō Ishinomori y las referencias a los ukiyo-e, o incluso a algunos de los seres humanos repugnantes y retorcidos de Hideshi Hino. Las caras redondeadas y muy sintéticas -especialmente la de Sadbøi (el protagonista) — se acercan a las de los héroes de los primeros thrillers de Tatsumi. El rostro de Sadbøi opta por un diseño icónico y minimalista, casi similar a un emoji, de forma que concentra casi todos los matices emocionales en los ojos y en los símbolos y otros elementos paralingüísticos.

Berliac camina por una fina línea entre la apropiación «estilo bellas artes» y el compromiso pleno con la creación de una espacio de encuentro coordinado entre el «manga clásico» y lo que creo es un increíble despliegue de cómics de autor de la escena argentina, y más allá [...]. Sin embargo, las decisiones de Berliac son bastante específicas. La composición de sus páginas, con retículas muy simples y excepciones teatrales, una figuración muy sintética, a veces hasta el extremo, las transiciones y la ambientación, sus temas de inspiración social, e incluso un uso acertado de la onomatopeya, recuerdan a una constelación muy coherente de artistas de los años 60 y 70. Estoy pensando en el grupo conocido como «12-3», formado por Shin'ichi Abe, Oji Suzuki, y Masuzou Furukawa, así como Yoshiharu Tsuge y Seiichi Hayashi. ${ }^{36}$

35 Berliac. «Ultimately Gaijin manga», en Facebook, 28 mayo 2018, 3.44 p.m.

36 Moura, P. «Sadbøi. Berliac (Canicola)», en Yellow Fast \& Crumble, 5 de febrero de 2020. Disponible en: yellowfastcrumble.wordpress.com/2020/02/05/sadboi-berliac-canicola/ 
Sadbøi es una novela gráfica monocroma, pero eso no significa que sea en blanco y negro. Al igual que muchas otras obras de Berliac, Sadbøi está impreso íntegramente en un tono índigo, de un modo similar a la impresión en tres colores (azul, entre otros) utilizada por los autores de gekiga a finales de los años cincuenta y sesenta. Además, para algunas ediciones internacionales (no así la edición española) Berliac insistió en elegir un papel y un material de cubierta - un papel de textura leñosa, muy delgado y ligeramente amarillento, y una sobrecubierta barata, flexible y con un acabado satinado- que se asemejan a las primeras publicaciones del gekiga. Sin embargo, esto último debe entenderse más como un homenaje que como una conexión real con el medio original; a pesar del parecido, la calidad del papel o la cubierta nada tienen que ver con los acabados pobres y de mala calidad presentes en las primeras obras del gekiga. En última instancia, el gaijin gekiga de Berliac se significa como una marca radicalmente diferente de otras etiquetas mangaescas, ya que no intenta imitar los estilos visuales actuales, sino que se basa en referencias de la escena manga de los años cincuenta y sesenta para construir su propio imaginario.

No obstante, esto no significa que Berliac sea ajeno al manga mainstream. En Sadbøi, al autor argentino emplea numerosos elementos convencionalizados e imagotipos propios del lenguaje visual del manga contemporáneo (y por ello reconocibles por los lectores habituales de manga japonés) entremezclados con otros recursos artísticos, sin que por ello caiga en la mímesis del manga más estandarizado y comercial. Al mismo tiempo, sus conexiones con un género histórico y un tanto anacrónico como es el gekiga, permiten que Berliac se aleje del debate sobre la copia que sí afecta a otros sellos mangaescos, al no referenciar directamente ningún estilo de manga más actual. Curiosamente, su conexión con el gekiga le permite apelar a un mayor número de lectores (incluyendo a aquellos que normalmente prefieren las novelas gráficas y, por extensión, el gekiga frente al manga contemporáneo) sin renunciar por ello a sus raíces mangaescas. Añade Moura:

En cualquier caso, la elección de Berliac hacia apropiaciones retóricas y estilísticas sigue algunas de las tendencias arraigadas en las prácticas contemporáneas de la historieta, que implican la creación de una tradición/canon privado, la auto-inmersión dentro de ese mismo campo, reconsiderar el poder político de esos textos-fuente en un nuevo contexto, por no hablar de un atajo preestablecido para ciertos efectos. ${ }^{37}$

\section{Conclusiones}

Aunque a menudo se privilegia la idea de los dos enfoques mencionados anteriormente (manga como estilo vs. manga «made in Japan») como polos antagónicos, no significa que se excluyan mutuamente, ya que un enfoque híbrido también es posible.

37 Idem. 
Kacsuk $^{38}$ explica cómo se entrelazan realmente ambas vertientes: cuando se trata del manga, los aspectos productivos han influido, históricamente, en el desarrollo estético de este medio, mientras que los elementos formales y la materialidad del manga han contribuido, igualmente y de manera significativa, a la consolidación del modelo de producción actual. Por otra parte, hay múltiples formas de referirse al manga y, si bien la categorización dicotómica sigue siendo una opción pertinente y determinante, no abarca necesariamente la totalidad del debate que, aun hoy en día, sigue muy presente en el ámbito académico. Quizás es el momento de, siguiendo la recomendación de Casey Brienza, ${ }^{39}$ preguntarse «Why do you care so much?» No en vano, dentro de la miríada de diferentes etiquetas mangaescas, lo que el debate «estilo vs. Japón» no tiene en cuenta es que el manga está siendo utilizado por autores y comunidades de aficionados de todo el mundo, no como un estilo ni como algo anclado en Japón, sino como un movimiento con una enorme base de fans, caracterizado por un gran sentido de pertenencia y un profundo conjunto de valores simbólicos e intangibles.

En este contexto, el gaijin gekiga de Berliac destaca por su planteamiento híbrido y profundamente transnacional, que mira hacia los autores japoneses de la década de 1950 y 1960 como punto de partida para la construcción de un lenguaje visual que, si bien posee irrenunciables raíces mangaescas, tiene una vocación internacional y radicalmente contemporánea.

38 Kacsuk, Z. Op. cit.

39 Brienza, C. Op. cit. 


\section{Bibliografía}

Abella, A. «La definición de la RAE para 'manga' indigna al mundo del cómic», en El Periódico, 22 de junio de 2012. Disponible en: http://www.elperiodico.com/ es/ocio-y-cultura/20120622/la-definicion-de-la-rae-para-manga-indigna-almundo-del-cómic-1961941

Berliac. Sadbøi. Barcelona, Roca Editorial (Sapristi Cómics), 2017.

— «Manga is Masturbation», en Asian Store Junkies. Vice, 2017. Disponible en: www.vice.com/en us/article/7xxdy4/manga-is-masturbation-todays-cómic-by-berliac-cómic

— UUltimately Gaijin manga», en Facebook, 28 de mayo de 2018, 3.44 p.m.

Berndt, J. «Traditions of Contemporary Manga (1): Relating Cómics to Premodern Art», en Signs. Studies of Graphic Narratives, n. 1 (2007), pp. 33-47.

— «Manga and 'Manga': Contemporary Japanese Cómics and their Dis/similarities with Hokusai Manga», en Jablonski, A. et al. (eds.) Civilization of Evolution, Civilization of Revolution, metamorphoses in Japan 1900-2000. Cracovia, Museum of Japanese Art and Technology, 2007, pp. 210-222.

-(ed.) Cómics Worlds and the World of Cómics: Towards Scholarship on a Global Scale. 1 vol. Global Manga Studies. Kioto, Kyoto Seika University (International Manga Research Center), 2010.

— «Facing the Nuclear Issue in a 'Mangaesque'Way: The Barefoot Gen Anime», en Cinergie, n. 2 (2012), pp. 148-162.

Boilet, F. «Manifiesto de la Nouvelle Manga», en Boilet.net, 2001. Disponible en: www.boilet.net/es/nouvellemanga manifeste $2 . \mathrm{html}$

Brienza, C. «Manga is not Pizza: The performance of ethno-racial authenticity and the politics of American anime and manga fandom in Svetlana Chmakova's Dramacon", en Brienza, C. (ed.) Global Manga: "Japanese" Cómics without Japan? Londres, Ashgate, 2015, pp. 95-113.

Brunner, V. «Lastman, le manga remixé french touch» en Les Inrockuptibles, 1 de mayo de 2014. Disponible en: http://www.lesinrocks.com/2014/05/01/livres/ lastman-manga-remixe-french-touch-11501782/ 
Burns, P. «An Apology», en Drawn E Quarterly, 2 de junio de 2017. Disponible en: www.drawnandquarterly.com/blog/2017/06/apology

Holmberg, R. «An Introduction to Gekiga, 6970 A.D.». The Comics Journal, 24 de abril de 2011. Disponible en: www.tcj.com/an-introduction-to-gekiga-6970-a-d/3/

Hernández Ranza, O. «Nouvelle Manga, mon amour. Reflexiones sobre la narración gráfica de historias cotidianas», en Ballaterra Journal of Teaching and Learning Language and Literature, n. ${ }^{\circ} 2$ (2010).

Horrocks, S. Mercurial Blonde, 21 de abril de 2015. Disponible en: mercurialblonde.tumblr.com/post/117018318913

Kacsun, Z. «Re-Examining the 'What is Manga' Problematic: The Tension and Interrelationship between the Style Versus Made in Japan Positions», en Arts, Volume 7, Issue 3 (2018).

LE SAux, L. «BD : Lastman, un manga à la française de Vivès, Balak et Sanlaville», en Télérama, 29 de mayo de 2013. Disponible en: www.telerama.fr/livre/bd-lastman-un-manga-a-la-francaise-de-vives-balak-et-sanlaville,96613.php

Moneyron, J. «kuš! Aesthetics», en The Comics Journal, 27 de octubre de 2016. Disponible en: http://www.tcj.com/kus-aesthetics/

Moura, P. «Sadbøi. Berliac (Canicola)», en Yellow Fast $\Xi$ Crumble, 5 de febrero de 2020. Disponible en: yellowfastcrumble.wordpress.com/2020/02/05/sadboi-berliac-canicola/

— «Desolation.exe. Berliac (Low Spirits)», en Lerbd, 21 de junio de 2016. Disponible en: lerbd.blogspot.com/2016/06/desolationexe-berliac-low-spirits.html

Parkas, V. «Berliac, el dibujante de cómic que reivindica el robo cultural como esencia creativa», en PlayGround, 14 de noviembre de 2017. Disponible en: http:// www.playgroundmag.net/lit/Berliac-el-chico-triste-que-hizo-de-la-apropiacion-cultural-una-forma-de-empoderamiento 23343930.html

— «Unas declaraciones transfóbicas del dibujante Berliac le han valido el odio de medio internet», en PlayGround, 8 de junio de 2017. Disponible en: www. playgroundmag.net/lit/Holocausto-Berliac-transfobia-cancelaciones-editoriales $22594415 . \mathrm{html}$ 
Romero Leo, J. «La estética de la Nouvelle Manga. La reivindicación de lo cotidiano en la nueva novela gráfica franco-japonesa», en $\mathrm{CuC}$ o, Cuadernos de cómic n. ${ }^{\circ} 8$ (2017), pp. 83-101. Disponible en: http://cuadernosdecomic.com/docs/revista8/La estetica de la Nouvelle Manga.pdf

Stewart, R. "Manga as schism: Kitazawa Rakuten's resistance to 'Old-Fashioned'Japan», en Berndt, J. y Kümmerling-Meibauer, B. (eds.) Manga's Cultural Crossroads. Londres, Routledge, 2013, pp. 27-49.

Stıvè, V. «Berliac e la scena Gaijiin Manga dell'antologia Kuš! \#25», en Fumetto Logica, 28 de febrero de 2017. Disponible en: www.fumettologica.it/2017/02/berliac-intervista-kus-gaijin-manga/2/

Yoкоуама, Y. World Map Room. s.1., PictureBox, 2013. 